\title{
Scaling and glassy dynamics in the relaxation of dislocation systems
}

\author{
Ferenc F. Csikor ${ }^{* \dagger}$ and Michael Zaiser \\ The University of Edinburgh, Centre for Materials Science and Engineering, The King's \\ Buildings, Edinburgh EH9 3JL, United Kingdom \\ †E-mail: Csikor@metal.elte.hu \\ ${ }^{\ddagger} E$-mail: M.Zaiser@ed.ac.uk
}

\begin{abstract}
The relaxation of initially random systems of infinite, straight, parallel edge dislocations is studied in single slip. A relaxation model is developed based on the gradual extinction of initial density fluctuations. Exponents of the predicted power law evolution for the mean value of different powers of dislocation velocity are compared to discrete dislocation dynamics simulations. A satisfying match is found in the theoretical limit of large multipole formation rates and a weak coupling between mobile excess dislocations and background multipoles.
\end{abstract}

International conference on Statistical Mechanics of Plasticity and Related Instabilities August 29-September 2, 2005

Indian Institute of Science, Bangalore, India

\footnotetext{
* Speaker.
} 


\section{Introduction}

Since its introduction nearly 20 years ago, discrete dislocation dynamics (DDD) simulation, i.e. the numerical integration of the equations of motion of crystal dislocations, has become a standard tool for studying the plastic properties of crystalline materials on the sub-micron scale. To mimic the incidental initial defect configuration in customary experimental samples, initial conditions of DDD simulations usually consist of a random dislocation configuration, relaxed in the absence of externally applied loads. Since only the outcome of this procedure is relevant to the subsequent simulation, the dynamical properties of the relaxation process are still largely unexplored. The two dimensional case of infinite, straight, parallel edge dislocations is particularly interesting because of the significant reorganization and short range pattern formation during the relaxation process [1]-4]. Only recently has a numerical study with 1024 such dislocations revealed a slow, glassy dynamics in the initial relaxation stage, marked by clean power law relaxation of several measurables like the average velocity or the total elastic energy, with exponents -0.90 and -0.60 , respectively [5]. This non exponential relaxation clearly hints at some kind of a collective behavior behind the relaxation process.

The present paper aims to enhance these studies by introducing a model of the relaxation of random dislocation arrangements and testing some of its predictions in a simple numerical setting. In Section 2, the mental frame of the model is set up based on a chemical analogy. This chemical model is then applied to the dislocation case. In Section 3, model predictions are systematically compared to the results of numerical simulations. Finally, Section 4 gives a summary and outlines future directions.

\section{Theory}

The driving force behind the relaxation of two dimensional dislocation systems is to decrease the total elastic energy of the embedding crystal through screening the long range stress fields of individual dislocations. This is analogous to the well known screening of the electric field in charge carrying fluids such as ionized gases and conduction electrons in metals [ $₫$ ]. The screening length in the dislocation case was found to be in the order of the average dislocation spacing [2]. On the microscopic level, screening manifests itself as the formation of dislocation multipoles with zero net Burgers vectors [1, 3]. A similar picture emerges from the work of Wilkens [6] who proved that i) the elastic energy density of a random dislocation system diverges logarithmically with the system size and that ii) this divergence can be canceled by restricting density fluctuations at short distances in a certain way (restricted random distribution). Point iii) is equivalent to a special kind of pair correlations or, in other words, a special kind of screening.

From the dynamics point of view, the first emerging question is the characterization of the build up process of a single multipole. To this end, two well known features of individual dislocations are recalled. i) The elastic stress field generated by a straight dislocation is inversely proportional to the distance from the dislocation line (at least at distances larger than the core radius). ii) The dynamics of dislocations is often overdamped, i.e. their velocity is a function of the stress acting on them. These two properties hint at a two stage build up step for multipoles: glide motion of an 
external dislocation with gradually increasing velocity, followed by a (relatively quick) trapping event at an equilibrium position inside the multipole.

The above two steps bear some similarity with the general features of chemical processes, namely that reactants undergo periods of Brown motion before entering into a reaction event. In this analogy, moving dislocations (and possibly multipoles) play the role of reactant molecules and low mobility multipoles the role of the products of the chemical reaction. To exploit this chemical analogy, we restrict the present analysis to the single slip of infinite, straight, parallel edge dislocations, where the two possible Burgers vectors represent two kinds of reactants. It is appropriate to note here that, in spite of the overly simplified dislocation geometry considered, the essence of the following analysis is built onto general features of dislocation systems, extending its validity to more complex slip geometries, too.

\subsection{Kinetics of a bimolecular chemical reaction}

In the above spirit, this section reviews the reaction kinetics of the bimolecular synthesis reaction

$$
\mathrm{A}+\mathrm{B} \rightarrow \mathrm{C} .
$$

The presentation follows the excellent work of Ovchinnikov and Zeldovich [7]. We consider an equal initial density of the reactants,

$$
\rho_{\mathrm{A}}^{0}=\rho_{\mathrm{B}}^{0} .
$$

Because of the stoichiometry of reaction (2.1), this equality is an invariant of the reaction. Furthermore, reaction (2.1) is "turned on" at a random arrangement of reactant molecules A and B at time $t=0$.

According to Ovchinnikov and Zeldovich, reaction (2.1) started from initial condition (2.2) proceeds in three consecutive stages.

Stage 1 comprises those molecular level reaction events which are accessible through the short range motion of reactants (short range being defined as not much more than the initial inter reactant spacing). Some of the reactant molecules can not react in this way because of initial density fluctuations present in the system. These "excess" molecules will react in later stages. The kinetics of stage 1 can be modeled with the well known rate equation

$$
\dot{\rho}_{\mathrm{A}}=\dot{\rho}_{\mathrm{B}}=-g \rho_{\mathrm{A}} \rho_{\mathrm{B}}
$$

where the bimolecular reaction rate $g$ is determined by the details of the reaction in question. For instance, for diffusion controlled reactions $g=4 \pi D r_{0}$ [8] where $r_{0}$ is the molecule radius and $D$ the relative diffusion coefficient. The solution of rate equation (2.3) with initial condition (2.2) takes the form

$$
\rho_{\mathrm{A}}=\rho_{\mathrm{B}}=\frac{\rho_{\mathrm{A}}^{0}}{1+g \rho_{\mathrm{A}}^{0} t} \text { at } t \geq 0, \quad \rho_{\mathrm{A}}=\rho_{\mathrm{B}}=\frac{1}{g t} \text { at } t \gg\left(g \rho_{\mathrm{A}}^{0}\right)^{-1} .
$$

Stage 2 of reaction (2.1) is entered when the number of excess molecules becomes dominant among the reactants. Excess molecules find each other via long range Brownian motion which defines the kinetics of this stage. For simplicity, we start the discussion in the 
limit of an infinitely large reaction rate $g \rightarrow \infty$. In this case, molecules A and B can not coexist at any time $t>0$ at any location, therefore the system can be fully characterized with the density difference $\kappa=\rho_{\mathrm{A}}-\rho_{\mathrm{B}}$. I.e., in regions with only molecules A $\rho_{\mathrm{A}}=\kappa$ and $\rho_{\mathrm{B}}=0$, otherwise $\rho_{\mathrm{A}}=0$ and $\rho_{\mathrm{B}}=-\kappa$. The dimensions of regions with $\kappa>0$ and $\kappa<0$ are determined by the diffusion length $L=\sqrt{D t}$. To predict the evolution of excess molecule densities, first the initial spatial fluctuation spectrum of $\kappa$ should be determined. It approximately obeys the Poissonian distribution, i.e. $\left\langle\left(\kappa^{0} V\right)^{2}\right\rangle \approx \rho_{\mathrm{A}}^{0} V=\rho_{\mathrm{B}}^{0} V$ for any region with volume $V$. On the other hand, the smallest region size still not affected by diffusion evolves as $V(t) \propto L^{3} \propto(D t)^{\frac{3}{2}}$. The combination of these two ideas leads to

$$
2 \rho_{\mathrm{A}}=2 \rho_{\mathrm{B}}=\langle|\kappa|\rangle \approx\left(\frac{\rho_{\mathrm{A}}^{0}}{V(t)}\right)^{\frac{1}{2}} \propto \frac{\left(\rho_{\mathrm{A}}^{0}\right)^{\frac{1}{2}}}{(D t)^{\frac{3}{4}}}
$$

(see [7] for details). This formula only makes sense when the diffusion length $L$ is larger than the initial intermolecular distance $\left(\rho_{\mathrm{A}}^{0}\right)^{-\frac{1}{3}}$. Another, but upper, time limit is marked by $L$ reaching system size $L_{s}$. It is also worth noting that the $t^{-\frac{3}{4}}$ decay in Eq. (2.5) is slower than the $t^{-1}$ extinction law of stage 1 (see Eq. (2.4)). Coming back to finite values of $g$, this difference implies a crossover between stage 1 and stage 2 behavior at $t \approx \frac{D^{3}}{\left(\rho_{A}^{0}\right)^{2} g^{4}}$.

Stage 3 steps in when the diffusion length $L$ reaches the system size $L_{S}$. Being irrelevant to the following, its discussion is omitted here.

\subsection{Density fluctuation dominated stage of the relaxation of dislocation systems}

As discussed above, the relaxation of two dimensional random dislocation configurations and the kinetics of chemical synthesis reactions are parallel to some extent. In this section we will adapt the concepts of Section 2.1 to the relaxation of $N$ infinite, straight, parallel edge dislocations in a single slip geometry. We start with formulating the dynamics problem of the dislocation system in question and proceed with working out two variants of a relaxation model, with a difference in the bonding strength between mobile excess dislocations and background multipoles.

We start the formulation by choosing the $z$ axis of a Cartesian coordinate system in the line direction of dislocations and the $x z$ plane of the same system parallel to their slip plane. In the following, dislocation $i(i=1 \ldots N)$ will be characterized with its intersection point with the $x y$ plane $\vec{r}_{i}$ and its Burgers vector $\vec{b}_{i}=s_{i} b \vec{e}_{x}$ where $s_{i}= \pm 1$ is the sign of the $i$ th dislocation, $b$ is the common magnitude of the Burgers vectors of dislocations in the system and $\vec{e}_{x}$ is the unit vector pointing in the $x$ direction. To get a smooth evolution of the total elastic energy ${ }^{1}$, we take the dislocation number $N$ conserved (to avoid energy jumps associated with multiplication and annihilation events). Furthermore, we use a vanishing net Burgers vector $\sum_{i=1}^{N} s_{i}=0$ (obligating $N$ to be even). Dislocation climb is disregarded and overdamped dislocation glide is assumed with a linear stress velocity rule. On these conditions, the equation of motion takes the form

$$
\frac{1}{\chi} \dot{x}_{i}=\sum_{j \neq i} b s_{i} s_{j} \tau_{\text {int }}\left(\vec{r}_{i}-\vec{r}_{j}\right)+b s_{i} \tau_{\mathrm{ext}}
$$

\footnotetext{
${ }^{1}$ A detailed analysis of the evolution of the total elastic energy is left to a subsequent paper.
} 
where $\chi$ denotes dislocation mobility, $\tau_{\text {int }}(\vec{r})$ the $x y$ component of the elastic stress in position $\vec{r}$ generated by a positive dislocation in the origin of the coordinate system and $\tau_{\text {ext }}$ the $x y$ component of the externally applied stress. In an infinite embedding crystal, $\tau_{\text {int }}(\vec{r})$ takes the form

$$
\tau_{\text {int }}(\vec{r})=G b \frac{x\left(x^{2}-y^{2}\right)}{\left(x^{2}+y^{2}\right)^{2}}
$$

where $G$ denotes the shear modulus of the crystal. Note that the elastic dislocation-dislocation interaction described by Eq. (2.7) is inversely proportional to $|\vec{r}|$ in the entire crystal space (i.e. the formula assumes a point like dislocation core) and has a strong angular dependence. In the present paper the external stress $\tau_{\text {ext }}$ is always zero; the case $\tau_{\text {ext }} \neq 0$ is relegated to a subsequent paper.

For clarity reasons, dimensionless coordinates will often be used in the following. Eqs. (2.6) and (2.7) only restrict dimensionless physical units up to an arbitrary length $l$. The choice $l=\rho^{-\frac{1}{2}}$ will be used below, yielding dimensionless length and time coordinates $x^{\prime}=x \sqrt{\rho}$ and $t^{\prime}=t \rho \chi G b^{2}$, respectively. As a convention, dimensionless quantities will be denoted by an apostrophe.

Consider now the relaxation of a random dislocation configuration. As already mentioned, this process can be viewed as the gradual formation of strongly bound multipoles from the initial individual dislocations. In line with the chemical case, three relaxation stages may be hypothesized.

Stage 1 is characterized by rapid, short range dislocation motion, whereby the bulk of dislocations gets trapped in multipoles. However, the Burgers vectors of some "excess" dislocations, stemming from density fluctuations in the initial configuration, can not be locally compensated. These excess dislocations compensate the Burgers vectors of each other during the subsequent

Stage 2 via long range glide motion. As in the chemical case, the dynamics of stage 2 can be captured through the evolution of the typical size $L$ of regions containing only excess dislocations with the same sign. The long range glide motion governing this evolution proceeds above a background of compensated multipoles. It is not obvious to what extent the moving excess dislocations drag the background multipoles with themselves. Therefore, the two extremes are worked out and compared to simulation results in the following. Similarly with the chemical case, one expects the onset of stage 2 when the region size $L$ reaches the average dislocation spacing (supposing a fast enough multipole formation in stage 1). Similarly,

Stage 3 is entered when $L$ reaches the system size $L_{s}$.

In the following, the dynamics of the density fluctuation dominated stage 2 will be worked out in the above two extreme cases. First the evolution equation of region size $L$ is derived. Based on the solution $L(t)$, evolution of the moments of dislocation velocity is predicted with the aim of comparing them to numerical simulations (see Section B).

\subsubsection{Free-flight model}

The case of freely flying excess dislocations only differs from the chemical case in the dynamics of excess particles (dislocation glide instead of Brownian motion). The modified stage 2 behavior is treated here in a simple scaling framework. 
As discussed above, during stage 2, excess dislocations with the same sign form regions of typical size $L$. A typical excess dislocation will then be attracted towards multipole formation by an excess dislocation in the neighboring region. The typical distance between these two dislocations is proportional to $L$, leading to a typical excess dislocation velocity proportional to $\frac{1}{L}$. By identifying the growth rate of region size with dislocation velocities (just like in the chemical case), one arrives at

$$
\frac{d L^{\prime}}{d t^{\prime}} \sim \frac{1}{L^{\prime}} \quad \Rightarrow \quad L^{\prime} \sim \sqrt{2 t^{\prime}}
$$

As can be seen, the evolution exponent $\frac{1}{2}$ of $L^{\prime}$ coincides with that in the chemical case. Eq. (2.8) only makes sense when the dimensionless region size $L^{\prime}$ is between the average dislocation distance 1 and the system size $L_{s} \sqrt{\rho}$. Assuming a large enough reaction rate for multipole formation, this leads to the following time constraints for stage 2 behavior:

$$
\frac{1}{2}<t^{\prime}<\frac{1}{2} \rho L_{s}^{2}
$$

While calculating the $n$th moment of dislocation velocity, $\left\langle\left|v^{\prime}\right|^{n}\right\rangle$, we neglect the motion of multipoles, so that $\left\langle\left|v^{\prime}\right|^{n}\right\rangle \sim E v^{\prime n}$ where $E$ is the concentration of excess dislocations and $v^{\prime}=$ $d L^{\prime} / d t^{\prime}$ the typical excess dislocation velocity. As in the chemical case, $E\left(t^{\prime}\right)$ is equal to the initial excess dislocation concentration in a region with size $L^{\prime}\left(t^{\prime}\right)$ since density fluctuations below $L^{\prime}\left(t^{\prime}\right)$ are already smoothed out. This leads to $E=\sqrt{L^{\prime 2}} / L^{\prime 2}=1 / L^{\prime}$ and, with Eq. (2.8), finally to

$$
\left\langle\left|v^{\prime}\right|^{n}\right\rangle \sim E v^{\prime n}=\frac{1}{L^{\prime}} \frac{1}{L^{\prime n}} \sim \frac{1}{\left(2 t^{\prime}\right)^{\frac{n+1}{2}}} .
$$

\subsubsection{Dragged multipole model}

Consider now the case when excess dislocations drag background multipoles with themselves. Since each region contains only excess dislocations with the same sign, this leads to regions moving as rigid blocks. This has three consequences. i) Excess dislocation mobility is reduced proportional to the concentration of excess dislocations, implying $v^{\prime} \sim 1 / L^{\prime 2}$. ii) Since rigid regions can not inter-penetrate, the displacement needed to join two charged neighbors reduces from $L^{\prime}$ to 1 (the average dislocation distance). iii) Such an event leads to a region size enhancement by $\Delta L^{\prime} \sim L^{\prime}$. All this leads to

$$
\frac{d L^{\prime}}{d t^{\prime}} \sim \frac{\Delta L^{\prime}}{\frac{1}{v^{\prime}}} \sim \frac{1}{L^{\prime}} \quad \Rightarrow \quad L^{\prime} \sim \sqrt{2 t^{\prime}}
$$

As can be seen, region size evolution, and so the time constraints of stage 2 , too, will be equal to the free-flying case.

On the other hand, the evolution of the moments of dislocation velocity will differ, since now all dislocations are in motion and with a typical velocity different from the free-flight case. Quantitatively this leads to

$$
\left\langle\left|v^{\prime}\right|^{n}\right\rangle \sim v^{\prime n} \sim\left(\frac{1}{L^{\prime 2}}\right)^{n} \sim \frac{1}{\left(2 t^{\prime}\right)^{n}} .
$$

As can be seen, the velocity exponents predicted by the two models differ for orders $n \neq 1$. 


\section{Numerical simulations}

\subsection{Simulation method}

To test the predictions of the two relaxation models in Sec.2.2.1 and Sec.2.2.2, we performed discrete dislocation dynamics simulations of the relaxation of initially random dislocation systems. To this end, the two dimensional single slip geometry described in Sec. 2.2 was confined to a square shaped simulation area with sides parallel to the $x$ and $y$ axes and side length $L_{s}$. Periodic boundary conditions were used both for dislocation kinematics and interactions (the method of the latter was taken from [9]). Eq. (2.6) was solved with an adaptive step size 4.5th order Runge-Kutta-Fehlberg method. As mentioned earlier, in the simulations the dislocation number was kept constant, i.e. neither multiplication nor annihilation was allowed, to avoid discontinuities in the evolution of the elastic energy. To avoid the prohibitively small time steps caused by narrow dipoles, the few narrowest ones were removed from the system and relaxed to their equilibrium "by hand". The number of removed dipoles was adjusted to cause no observable changes on the simulation results. All simulations were started from random configurations of an equal number of positive and negative sign dislocations.

\subsection{Simulation results and discussion}

As expected, individual relaxation simulations showed strongly fluctuating activity in the form of a series of dislocation avalanches with decreasing amplitudes (for a recent review on intermittent plastic behavior see [10]). To get access to the smooth behavior described in Sec. 2.2, averages of large numbers of independent simulations were calculated. Results with 16, 32, 48 and 64 dislocations (i.e. with system sizes $L_{s}^{\prime}=4,4 \sqrt{2}, 4 \sqrt{3}$ and 8 ) are presented below, with a statistics of $10^{4}-10^{5}$ in each case. Simulation of larger systems is in progress.

Figure 1 displays the evolution of the mean square velocity $\left\langle v^{2}\right\rangle$ at different system sizes, along with the predictions of the free-flight and multipole drag models. As can be seen, the multipole drag exponent significantly differs from the simulation results while the free-flight exponent almost agrees with them. There is a small difference, though, although its character is hard to establish at the current simulation statistics. An end of the scaling region can not be identified either. On the other hand, the start of the scaling region is clearly at $t^{\prime} \approx 0.4$ for all system sizes which agrees well with the predicted $\frac{1}{2}$ for the case of a large reaction rate $g$ (see Eq. (2.9)). This is a strong argument in favor of the consistency of the model with the numerics. It is also notable that results for different system sizes can be collapsed in the entire time range by rescaling $\left\langle v^{2}\right\rangle$ with $\ln \left(L_{s}^{\prime}\right)$. The origin of this scaling property is at this point unclear. It might be connected to the logarithmic divergence of the elastic energy of excess dislocations with the system size or perhaps to the finite simulation box size.

The evolution of higher order velocity moments $\left\langle|v|^{3}\right\rangle$ and $\left\langle v^{4}\right\rangle$ was also calculated from the available numerical data. Although their statistical noise is even larger than that of $\left\langle v^{2}\right\rangle$, they clearly support the free-flight model against the multipole drag model.

As the last result in this section, the evolution of the average velocity $\langle|v|\rangle$ is presented in Figure 2. The average velocity is close to a power law function with an exponent $\approx-0.86$ for over a decade of time for the largest system size. This is close to the exponent -0.90 found earlier on the initial part of the scaling regime in a larger system with $L_{s}^{\prime}=32$ []. The difference might be due to 


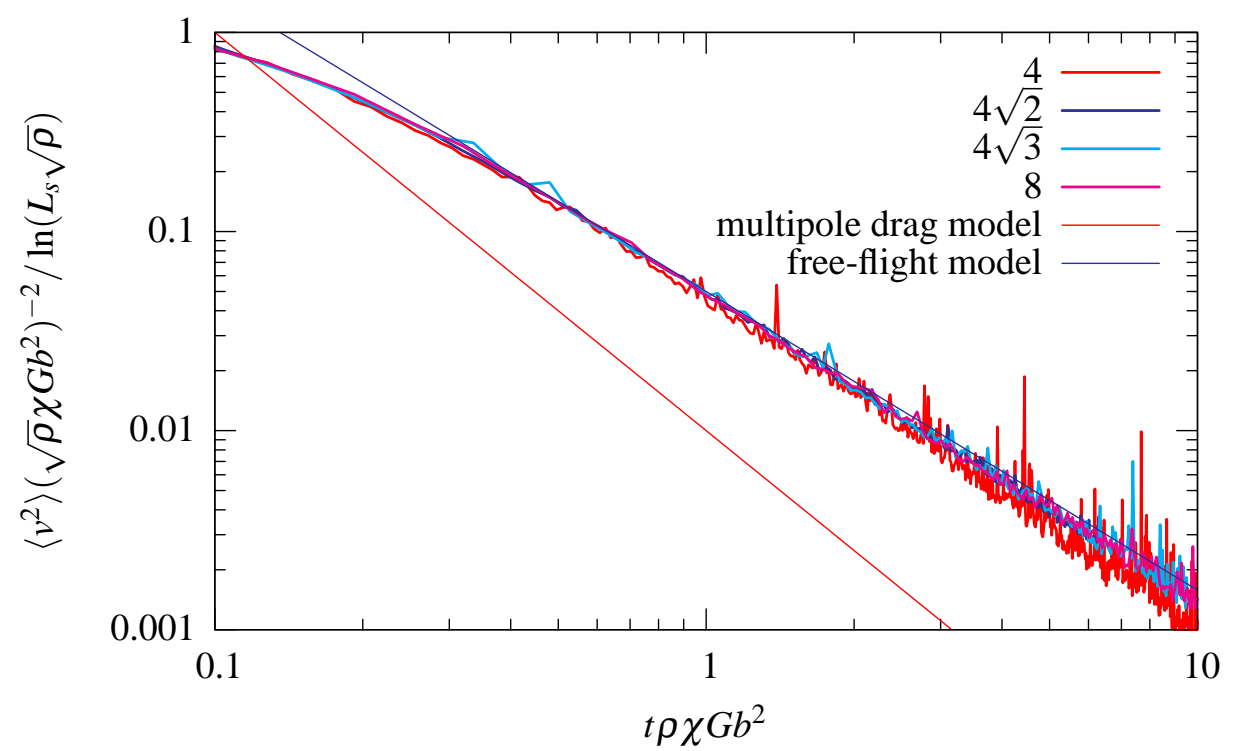

Figure 1: Numerically observed evolution of $\left\langle v^{2}\right\rangle$ for different system sizes (see legend) and model functions proportional to the predictions of the multipole drag and free-flight models. $\left\langle v^{2}\right\rangle$ values are collapsed with the scaling function $\ln \left(L_{s}^{\prime}\right)$. All units are dimensionless.

non-power law corrections (see next paragraph). It is also clearly seen that the common prediction of the two models is not far from the numerical curves. The difference between the predictions of the models and the numerical results is analyzed below in detail. Still in Figure 2, the beginning and end of the scaling region is clearly visible. In line with the prediction in Eq. (2.9), the former is independent of the system size while the latter is an increasing function of it. Again, a logarithmic factor is able to collapse the data, although its form $\ln \left(5 L_{s}^{\prime}\right)$ is slightly different from the case of $\left\langle v^{2}\right\rangle$.

To analyze the difference between the numerical results and the predictions of the models, their ratio is displayed in Figure 3. As can be seen, a logarithmic correction is able to describe the observed difference ${ }^{2}$ which might be rationalized by completing the equation of motion in Eq. (2.8) (or Eq. (2.11) ) with a logarithmic factor of the region size $L^{\prime}$. This modification might also have the potential explaining the deviation between the numerics and the current model for $\left\langle v^{2}\right\rangle$.

Besides this, the onset of stage 2 can be clearly identified in Figure 3 to be at $t^{\prime} \approx 0.4$ for all system sizes which is consistent with the numerical results for $\left\langle v^{2}\right\rangle$ and the models, too, assuming a large value of reaction rate $g$. Stage 2 -stage 3 transition times can also be read from Figure 3 and their values are consistent with the proportionality to $\rho L_{s}^{2}$ predicted by the models (see Eq. (2.9)). Even the prefactor to $\rho L_{s}^{2}, \approx 0.15$, does not fall too far from the predicted $\frac{1}{2}$. These findings strongly support that the model is relevant in the numerically studied time regime.

\section{Summary and future directions}

The relaxation of initially random systems of infinite, straight, parallel dislocations was stud-

\footnotetext{
${ }^{2}$ A power law correction with a small exponent is able to fit the difference, too, but this form is harder to rationalize.
} 


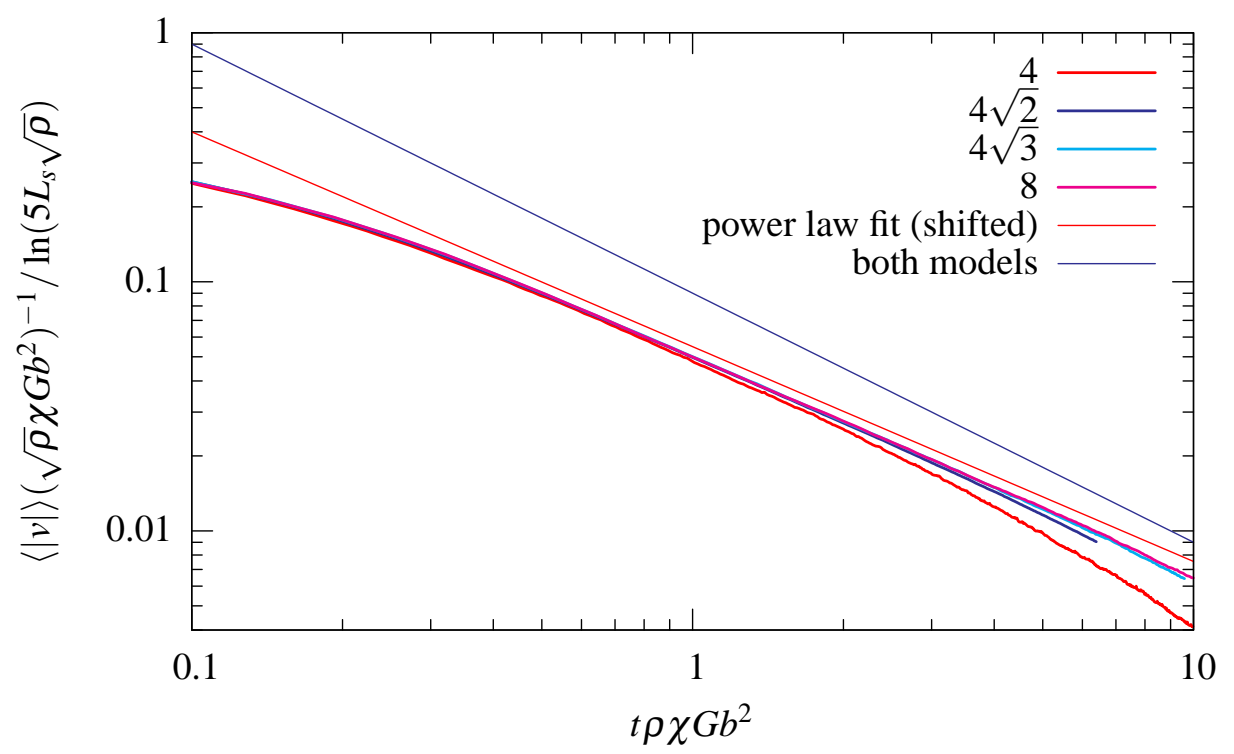

Figure 2: Evolution of $\langle|v|\rangle$ with the common prediction of the two models and a power law fit to the numerical curve at the largest system size. Simulation results are collapsed using function $\ln \left(5 L_{s}^{\prime}\right)$. All units are dimensionless.

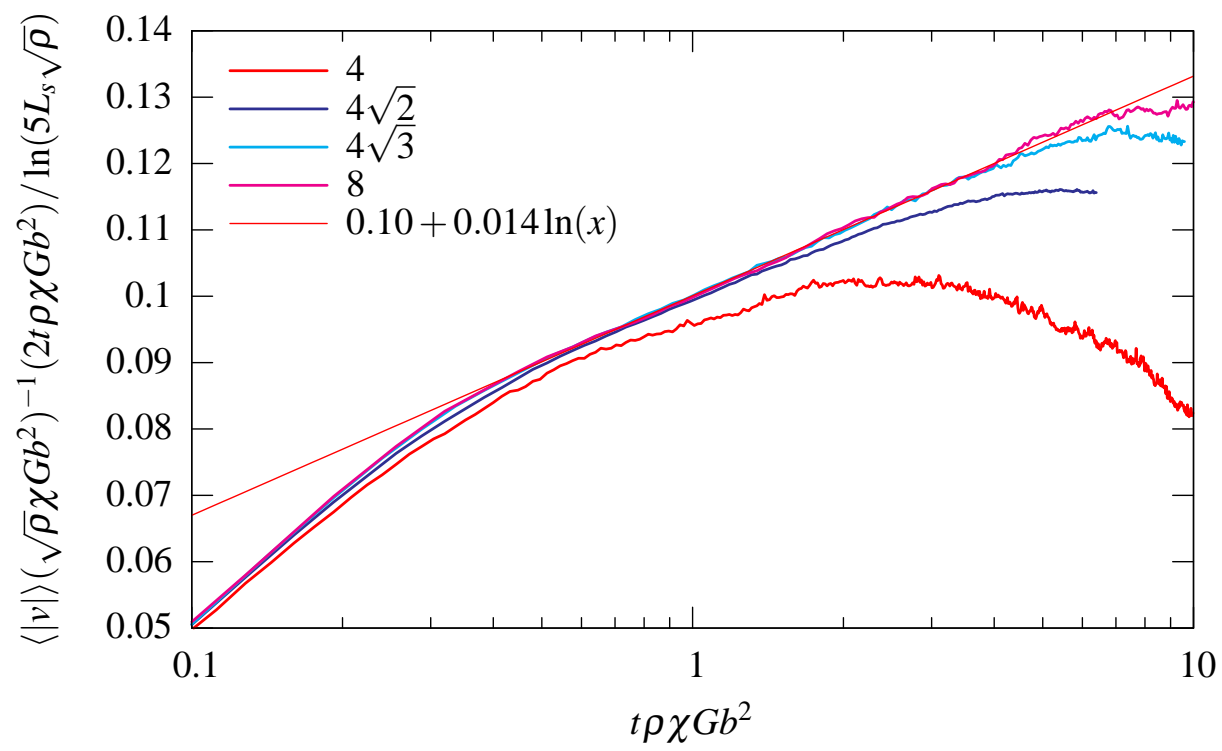

Figure 3: Ratio of the numerical values of average velocity and the common prediction of the models, together with a logarithmic fit to the stage 2 part of the numerical curves. 
ied. Noting kinematic similarities between chemical synthesis reactions and multipole formation during dislocation relaxation, the theory for the kinetics of the chemical case was adapted to the relaxation process in the special case of single slip. The central element of both models is the gradual extinction of initial density fluctuations at increasing length scales. The adapted model predicts a slow, glassy, power law evolution for the moments of the velocity distribution. The obtained exponents are in line with discrete dislocation dynamics simulations in the limit of weak coupling between mobile excess dislocations and background multipoles and a sufficiently large multipole formation rate.

Besides these results, plenty of questions are still open. Numerical simulations revealed a weak (presumably logarithmic) deviation in the evolution of the moments of the velocity distribution from the predictions of the simple scaling model. A weak (most likely also logarithmic) system size dependence also emerged. It is not clear at the moment if these deviations are intrinsic to the relaxation process or are the results of the specific boundary conditions used in the simulations. Going to larger simulated system sizes could certainly reveal more details of these deviations but hard computational limits inhibit the simulation of radically larger systems. Moving to $O(N)$ algorithms like particle-particle-particle-mesh [11, 12] or stochastic [13] methods might be an option, although the involved meshing of the simulation volume may give an inaccurate representation of region size $L$. Beyond numerical improvements, new questions like the evolution of elastic energy or the application of nonzero external stresses can be raised. We defer these topics to a subsequent publication.

\section{Acknowledgments}

Financial support of the European Community's Human Potential Programme under Contract No. MRTN-CT-2003-504634 [SizeDepEn] is gratefully acknowledged.

\section{References}

[1] H.Y. Wang, R. LeSar and J.M. Rickman, Analysis of dislocation microstructures: impact of force truncation and slip systems, Phil. Mag. A 78 (1998) 1195.

[2] M. Zaiser, M.C. Miguel and I. Groma, Statistical dynamics of dislocation systems: The influence of dislocation-dislocation correlations, Phys. Rev. B 64 (2001) 224102.

[3] F.F. Csikor and I. Groma, Probability distribution of internal stress in relaxed dislocation systems, Phys. Rev. B 70 (2004) 064106.

[4] I. Groma, G. Györgyi and B. Kocsis, Debye screening of dislocations, Phys. Rev. Lett., submitted.

[5] F.F. Csikor, B. Kocsis, B. Bakó and I. Groma, Numerical characterisation of the relaxation of dislocation systems, Mat. Sci. Eng. A 400-401 (2005) 214.

[6] M. Wilkens, Das mittlere Spannungsquadrat $\left\langle\sigma^{2}\right\rangle$ begrenzt regellos verteilter Versetzungen in einem zylinderförmigen Körper, Acta Metall. 17 (1969) 1155.

[7] A.A. Ovchinnikov and Ya.B. Zeldovich, Role of density fluctuations in bimolecular reaction kinetics, Chem. Phys. 28 (1978) 215.

[8] M. Smoluchowsky, Drei Vorträge über Diffusion, Brownsche Bewegung und Koagulation von Kolloidteilchen, Physik. Z. 17 (1916) 557. 
[9] B. Bakó, I. Groma, I. Mastorakos and E.C. Aifantis, Investigation of dislocation patterning by stochastic integration of dislocation trajectories, Model. Simul. Mater. Sci. Eng. 13 (2005) 671.

[10] M. Zaiser, Scale invariance in plastic flow of crystalline solids, Adv. Phys., submitted.

[11] H.Y. Wang and R. LeSar, O(N) algorithm for dislocation dynamics, Phil. Mag. A 71 (1995) 149.

[12] D.B. Barts and A.E. Carlsson, Order- $N$ method for force calculation in many-dislocation systems, Phys. Rev. E 52 (1995) 3195.

[13] B. Bakó and I. Groma, Stochastic $O(N)$ algorithm for dislocation dynamics, Model. Simul. Mater. Sci. Eng. 7 (1999) 181. 